\title{
Mulheres Submetidas à Quimioterapia e suas Funções Cognitivas
}

\author{
Camila Vasconcelos Carnaúba Lima \\ Universidade Federal de Alagoas, AL, Brasil.
}

\author{
Raner Miguel Ferreira Póvoa \\ Universidade Federal de Alagoas, AL, Brasil.
}

Resumo: Atualmente, o câncer representa um grande desafio científico. A quimioterapia tem sido a alternativa de tratamento de maior perspectiva para o combate ao câncer. Durante esse percurso, alterações cognitivas podem ser acarretadas e, portanto, faz-se necessário a identificação destas, já que representam complicações neurológicas com alto índice de melhora quando tratadas. O objetivo desse trabalho delimitou-se a estudar as funções executivas em mulheres com câncer de mama submetidas à quimioterapia buscando identificar aspectos como flexibilidade cognitiva, impulsividade/controle inibitório, memória operacional, planejamento, tomada de decisão, categorização e fluência. Trata-se de uma revisão de literatura a partir de levantamento de publicações dos últimos cinco anos nas bases de dados PubMed, Lilacs, Pepsico, SciELO e BVS. Foram utilizados os seguintes descritores: avaliação neuropsicológica/neuropsychological evaluation, funções executivas/functions executive, quimioterapia/ chemotherapy e câncer de mama/breast cancer. Foram encontrados 18 artigos relacionados ao tema. Os estudos apontaram a existência de alterações no funcionamento executivo de mulheres submetidas à quimioterapia. Ressalta-se que essas pesquisas se diferenciaram entre si, no que se refere à caracterização da amostra e a métodos utilizados, o que demonstra pouca padronização da avaliação neuropsicológica desse domínio cognitivo. Conclui-se que os domínios cognitivos referentes às funções executivas se encontram mais prejudicados em mulheres com câncer de mama que receberam quimioterapia. Contudo, essas informações ainda são insuficientes, pois subdomínios das funções executivas precisam ser investigados com maior integralidade, a fim de se obter um maior panorama funcional. Sugere-se novos estudos acerca do rastreio das funções executivas.

Palavras-chave: Câncer de mama Quimioterapia, Funções executivas. 


\title{
Women Undergoing Chemotherapy and Their Cognitive Functions
}

\begin{abstract}
Currently, cancer is a major scientific challenge. Chemotherapy has been the most promising alternative treatment to combat cancer. Along the way, cognitive changes can occur and therefore it is necessary to identify these changes, as they represent neurological complications with high improvement when treated. The objective of this study was to study executive functions in women with breast cancer undergoing chemotherapy, seeking to identify aspects such as cognitive flexibility, impulsivity / inhibitory control, operational memory, planning, decision making, categorization and fluency. This is a literature review of publications of the last five years in PubMed, Lilacs, Pepsico, SciELO and BVS. The following descriptors were used: avaliação neuropsicológical neuropsychological evaluation, funções executivas/functions executive, quimioterapia/chemotherapy and câncer de mama/breast cancer. 18 articles related to the topic were found. The studies showed the existence of changes in the executive functioning of women undergoing chemotherapy. It is noteworthy that these studies differed among themselves as regards the characterization of the sample and the methods used, which shows little standardization of neuropsychological assessment of this cognitive domain. It is concluded that the cognitive domains related to executive functions are most affected in women with breast cancer receiving chemotherapy. However, this information is still insufficient because subdomains of the executive functions need to be studied in greater integrity in order to achieve greater functional landscape. It is suggested that new studies on the screening of the executive functions must be performed.
\end{abstract}

Keywords: Breast cancer, Chemotherapy, Executive functions.

\section{Mujeres Sometidas a Quimioterapia y sus Funciones Cognitivas}

Resumen: En la actualidad, el cáncer es un importante reto científico. La quimioterapia ha sido el tratamiento alternativo más perspicaz para combatir el cáncer. En el camino, cambios cognitivos pueden ser provocados, por lo que es necesario identificarlos, ya que representan complicaciones neurológicas con alta mejora al ser tratados. El objetivo de este trabajo se delimitó a estudiar las funciones ejecutivas en mujeres con cáncer de mama sometidas a quimioterapia buscando identificar aspectos como flexibilidad cognitiva, impulsividad / control inhibitorio, memoria operacional, planificación, toma de decisión, categorización y fluencia. Se trata de una revisión de literatura de publicaciones de los últimos cinco años en PubMed, Lilacs, PepsiCo, SciELO y BVS. Se utilizaron los siguientes descriptores: avaliação neuropsicológical neuropsychological evaluation, funções executivas/functions executive, quimioterapial chemotherapy y câncer de mama/breast cancer. Se encontraron 18 artículos relacionados con el tema. Los estudios demostraron la existencia de cambios en el funcionamiento ejecutivo de las mujeres sometidas a quimioterapia. Es de destacar que estos estudios difieren entre sí respecto a la caracterización de la muestra y los métodos utilizados, que muestra poca estandarización de la evaluación neuropsicológica de este dominio cognitivo. Se llegó a la conclusión de que los dominios cognitivos relacionados con las funciones ejecutivas son los más afectados en las mujeres con cáncer de mama que reciben quimioterapia. Sin embargo, esta información es todavía insuficiente porque los subdominios de las funciones ejecutivas deben ser estudiados en mayor integridad con el fin de lograr un mayor paisaje funcional. Se sugiere hacer nuevos estudios sobre la proyección de las funciones ejecutivas.

Palabras claves: Cáncer de mama, Quimioterapia, Las funciones ejecutivas. 


\section{Introdução}

O câncer é uma patologia acarretada pelo crescimento incontrolável de células anormais no organismo podendo, estas, invadir tecidos adjacentes e se espalhar - para outras partes do corpo - através da corrente sanguínea e do sistema linfático. Esse crescimento forma um tumor maligno caracterizado de maneira atípica, com formação de células anaplásicas (diferentes das do tecido normal) apresentando crescimento rápido destas com mitoses anormais e numerosas. A massa que compõe o câncer é pouco delimitada e localmente invasiva, podendo se infiltrar em tecidos adjacentes com metástase frequentemente presente (Instituto Nacional de Câncer, 2012). De vários tipos de câncer em mulheres, a mama corresponde à localização primária em $56,20 \%$ dos casos, sendo a maior em relação às outras (como de cólon e reto, útero, ovário etc). No Brasil, a estimativa do Instituto Nacional do Câncer - INCA (2016) prevê que 57.960 novos casos venham a surgir em 2016. Desconsiderando o câncer de pele não melanoma, esse é o tipo mais sofrido pelas mulheres nordestinas, contabilizando cerca de $38 \%$ dos casos de câncer nesse público. Estima-se que, em 2016, 11.190 (20\%) casos de câncer de mama sejam diagnosticados nessa região e, em Alagoas, estima-se cerca de 30 casos a cada 100 mil mulheres.

A quimioterapia tem sido a alternativa de tratamento de maior perspectiva para o combate ao câncer capaz de destruir pequenos focos de crescimento tumoral que se espalham pelo organismo e não podem ser acessadas via cirurgia ou radioterapia (Caponeto, \& Lange, 2008). No entanto, pesquisas em Neurociências têm demonstrado que esse tipo de tratamento pode acarretar alterações cognitivas. Em estudos anteriores, tais prejuízos foram associados à depressão, ansiedade ou fadiga, porém, pesquisas recentes - que controlam esses aspectos - mostram a permanência de alterações cognitivas em pessoas após serem submetidas à quimioterapia. Na verdade, observa-se que o funcionamento cognitivo dessas pessoas é reduzido, apesar de que, em alguns casos, permanece normal. E, quando alterado, pode afetar domínios cognitivos (Ahles, \& Saykin, 2007).

A ocorrência dessas alterações dependerá tanto do regime terapêutico quanto da vulnerabilidade particular do indivíduo. A quimioterapia, ainda, é responsável por déficits é responsável por déficits na reparação do ácido desoxirribonucleico (DNA), molécula que guarda a codificação genética dentro de cada célula. Estudiosos indicam danos oxidativos do DNA em mulheres com câncer de mama e problemas relacionados à replicação desta molécula no que se trata do encurtamento dos telômeros. Déficits neste último aspecto estão associados ao envelhecimento, ao Alzheimer, à predisposição do câncer e à mortalidade de idosos. Uma de suas causas, portanto, implica a submissão à quimioterapia (Ahles, \& Saykin, 2007).

Além disso, tanto a quimioterapia quanto fatores de desenvolvimento do câncer também podem estar associados ao aumento das citocinas, o que interfere direta ou indiretamente no desempenho cognitivo. Outro efeito colateral da quimioterapia atinge o catecol O-metiltransferase (COMT), um importante modulador da dopamina que interfere no funcionamento da memória e das funções executivas. A quimioterapia também pode reduzir taxas de estrogênio e testosterona sobre o funcionamento cognitivo. Nesse caso, as mulheres podem vivenciar uma menopausa abrupta, ou seja, antecipar seus sintomas no comprometimento cognitivo, principalmente, em termos de memória de trabalho (Ahles, \& Saykin, 2007). Por fim, numa pesquisa básica realizada por Hecktheuer (2009), alterações neurocomportamentais foram encontradas em ratos após a administração aguda da cisplatina, um determinado agente antineoplásico. Noutra pesquisa, camundongos apresentaram danos cognitivos após serem submetidos a apenas uma dose injetável de ciclofosfamida, comumente utilizado em tratamentos de câncer de mama (Reiriz, 2008).

Diante disso, o objetivo dessa revisão é levantar estudos de avaliação neuropsicológica especificamente do funcionamento executivo em mulheres com câncer de mama que receberam quimioterapia. A neuropsicologia é um ramo da neurociência que "avalia a função cerebral por meio de inferências do comportamento cognitivo, sensoriomotor, emocional e social do indivíduo", conforme Howieson e Lezak (2014, p. 41). Ainda de acordo com os autores, o processo avaliativo pode ser feito por meio de entrevistas, observação e aplicação de testes. Dados neuropsicológicos são indicadores de disfunção cerebral para muitas condições (Howieson, \& Lezak, 2014).

As funções executivas, domínio cognitivo delimitado nesse estudo, dizem respeito a um conjunto de habilidades que direcionam comportamentos a metas, avaliação de sua eficiência e adequação deles a fim de resolver problemas (Robbins, Weinberger, Taylor, \& 
Morris, 1996). Trata-se de realizações de tarefas cotidianas quando o indivíduo consegue estabelecer objetivos e traçar um plano de metas seguindo uma organização hierárquica facilitadora da execução, além de avaliar os resultados decorrentes disso, corrigir estratégias sem êxito e adotar novas rotas resolutivas. As funções executivas funcionam de maneira integrada, o que demonstra sua articulação com outros domínios cognitivos, como a atenção e memória. Falhas no funcionamento executivo podem dificultar a realização de atividades corriqueiras em pessoas com comprometimento cerebral adquirido ou com desenvolvimento anormal nessa área, como citam Malloy-Diniz, Paula, Sedó, Fuentes e Leite (2014).

Espera-se que a presente revisão possa levantar informações a fim de evidenciar o conhecimento acerca das funções executivas na área da Oncologia. Também espera-se fomentar discussão sobre o acompanhamento assistencial às mulheres com câncer de mama visando a uma quimioterapia integrada, incrementando a esse tratamento amplas possibilidades terapêuticas. Isto é, um acompanhamento de cuidados que considere, para além de efeitos colaterais sistêmicos e de sobrevida da doença, a qualidade de vida nas atividades diárias dessa usuária bem como em suas relações sociais e familiares.

\section{Método}

Foi realizada uma revisão de literatura, na qual buscou-se identificar publicações bibliográficas recentes que abordassem possíveis impactos da quimioterapia em pacientes mulheres com câncer de mama. Para tanto, foram acessados os bancos de dados: PubMed, Lilacs, Pepsico, SciELO e BVS. A busca abrangeu um período de 5 anos, de 2011 até 2016. Os termos de busca utilizados para localizar o material da pesquisa foram avaliação neuropsicológica/neuropsychological, funções executivas/functions executive, quimioterapia/ chemotherapye câncer de mama/ breast cancer.

A seleção dos artigos foi delimitada sob os seguintes critérios de inclusão: estudos de acesso aberto (sem valor financeiro a cobrar), pesquisas com participantes mulheres com câncer de mama submetidas à quimioterapia e com uso de recursos de avaliação neuropsicológica (testes, escalas e tarefas). Sendo assim, foram excluídos artigos repetidos, de revisão de literatura ou de meta-análise e pesquisas realizadas em animais ou sem relação com o câncer de mama e funções executivas. Também foram excluídos estudos cujo perfil exclusivo das participantes abrangia mulheres em pós-menopausa, idosas ou submetidas à terapia hormonal (para além da quimioterapia).

As publicações que continham os três termos no título, nas palavras-chave ou no seu resumo, foram separadas. Foi realizada, então, primeiramente, a leitura do resumo do artigo e, quando constatados os critérios de inclusão, sua leitura na íntegra.

A fim de examinar e sistematizar o material, foi feita, numa primeira etapa, uma caracterização dos estudos selecionados na qual foram destacadas as seguintes informações: a) Identificação dos trabalhos da amostra: autoria e fonte de acesso; b) Desenho e amostra - contendo principais informações no que se refere ao tipo de estudo (longitudinal ou transversal), número de participantes, perfis dos grupos participantes; c) Domínios cognitivos estudados e respectivos instrumentos de avaliação; d) Principais resultados referentes às funções executivas; e) Possíveis lacunas de estudos na área.

Após a caracterização dos estudos, as informações foram organizadas em tabelas. A seguir, discutiu-se acerca dos dados levantados dando ênfase na avaliação das funções executivas, domínio cognitivo de interesse do estudo em foco, utilizando-se a literatura científica existente sobre a temática.

\section{Resultados}

Após a busca, 51 artigos relacionados ao tema foram encontrados no PubMed e cinco na BVS. Nas demais bases de dados - Lilacs, Pepsico e SciELO -, nenhum estudo foi encontrado. Do total dos artigos levantados, apenas 18 deles foram compatíveis com os critérios de inclusão desse estudo e, portanto, selecionados para análise sendo caracterizadas na Tabela 1.

A maioria dos estudos explorou, além das funções executivas, outros domínios cognitivos e sintomas concomitantes ao processo da avaliação (Ando-Tanabe, 2014, Askren et al., 2014; Chen et al., 2013; 2014; Dumas, 2013; Ganz et al., 2003; Jansen, 2011; Kam et al., 2016; Kesler et al., 2011; 2013; Koppelmans et al., 2012; Lyon et al., 2016; McDonald et al., 2013; Miao et al., 2016; Park et al., 2015; Piccirillo, et al., 2015; Yao et al., 2016; Zheng et al., 2014). Apenas Kam et al. (2016), Miao et al. (2016) e McDonald et al. (2013) delimitaram-se à investigação e discussão aos aspectos relevantes do funcionamento executivo. 
Tabela 1

Identificação dos estudos selecionados, desenho e amostra dos estudos selecionados.

\begin{tabular}{|c|c|c|c|c|c|}
\hline Autoria & Periódico de publicação & $\begin{array}{l}\text { Local da } \\
\text { pesquisa }\end{array}$ & Desenho & $\begin{array}{l}\text { Tamanho } \\
\text { da amostra }\end{array}$ & $\begin{array}{c}\text { Intervalo } \\
\text { entre idade de } \\
\text { participantes }\end{array}$ \\
\hline Lyon, et al. (2016) & J Cancer Res Clin Oncol & EUA & Longitudinal & 77 & $21-71$ \\
\hline Yao et al. (2016) & $\begin{array}{c}\text { JInternational } \\
\text { Neuropsychological Society }\end{array}$ & Canadá & Transversal & 56 & $25-65$ \\
\hline Miao et al. (2016) & $\begin{array}{l}\text { European Journal of } \\
\text { Radiology }\end{array}$ & China & Longitudinal & 49 & $24-51$ \\
\hline Piccirillo et al. (2015) & Oncology & EUA & Transversal & 28 & $36-69$ \\
\hline Kam et al. (2016) & $\begin{array}{l}\text { Brain Imaging and } \\
\text { Behavior }\end{array}$ & Canadá & Transversal & 24 & $40-65$ \\
\hline Park, Bae, Jung, \& Jung (2015) & J Korean Acad Nurs & China & Longitudinal & 137 & $18-60$ \\
\hline Askren et al. (2014) & Breast Cancer Res Treat & EUA & Longitudinal & 97 & $30-71$ \\
\hline Zheng et al. (2014) & Breast Cancer Res Treat & China & Longitudinal & 1.059 & $20-75$ \\
\hline Ando-Tanabe et al. (2014) & Breast Cancer & Japão & Longitudinal & 38 & $32-82$ \\
\hline Chen et al. (2014) & Natl Med J China & China & Longitudinal & 79 & $26-64$ \\
\hline Chen et al. (2013) & Brain Research & China & Transversal & 186 & $26-64$ \\
\hline Dumas et al. (2013) & Brain Imaging Behav & EUA & Longitudinal & 9 & $42-71$ \\
\hline Ganz et al. (2003) & J Natl Cancer Inst & EUA & Transversal & 189 & $48-53$ \\
\hline Kesler et al. (2013) & Brain Imaging Behav & EUA & Transversal & 36 & $40-75$ \\
\hline $\begin{array}{l}\text { McDonald, Conroy, Smith, } \\
\text { West , \& Saykin (2013) }\end{array}$ & $\begin{array}{l}\text { Brain, Behavior, and } \\
\text { Immunity }\end{array}$ & EUA & Longitudinal & 79 & $29-70$ \\
\hline Koppelmans et al. (2012) & $\begin{array}{l}\text { Journal of Clinical } \\
\text { Oncology }\end{array}$ & Holanda & Longitudinal & 196 & $50-80$ \\
\hline Kesler, Kent, \& O’Hara (2011) & Arch Neurol & China & Transversal & 62 & $37-71$ \\
\hline $\begin{array}{l}\text { Jansen, Cooper, Dodd \& } \\
\text { Miaskowski (2011) }\end{array}$ & Support Care Cancer & EUA & Longitudinal & 91 & $18-65$ \\
\hline
\end{tabular}

Ademais, os estudos também variaram no uso de instrumentos de investigação. Apenas o teste Stroop, visando à avaliação de controle inibitório, foi o mais utilizado (Chen et al., 2013; 2014; Jansen et al., 2011; Kam et al., 2016; Koppelmans et al., 2012; Lyon et al., 2016; Miao et al., 2016; Yao et al., 2016; Zheng et al., 2014). É possível conferir essa variabilidade de métodos de avaliação na Tabela 2 .

A Tabela 3 apresenta os domínios cognitivos referentes às funções executivas - planejamento, impulsividade/controle inibitório, tomada de decisão, flexibilidade cognitiva, memória operacional, categorização e fluência, conforme Malloy-Diniz et al. (2014) - que foram investigados nos estudos levantados. Destaca-se a impulsividade/controle inibitório, sendo o componente de maior interesse pelas pesquisas (Chen et al., 2013; 2014; Jansen et al., 2011; Kam et al., 2016; Koppelmans et al., 2012; Lyon et al., 2016; Miao et al., 2016;
Yao et al., 2016; Zheng et al., 2014.). Por outro lado, apenas um estudo (Chen et al., 2013) avaliou o quesito de tomada de decisão e, nenhum deles, de planejamento.

Partindo para os resultados das pesquisas que aplicaram baterias de testes neuropsicológicos, a maioria dos subdomínios que correspondem às funções executivas mostraram-se alteradas em grupos de mulheres com câncer de mama em condição de quimioterapia (Chen e al., 2014; Ganz et al., 2003; Koppelmans, 2012; Lyon et al., 2016; Park et al., 2015; Yao et al., 2016; Zheng et al., 2014). Corroborando com esses dados, Askren et al. (2014), Dumas (2013), Kam et al. (2016), Kesler (2011; 2013), Miao et al. (2016), McDonald et al. (2013) e Piccirillo et al. (2015), ao examinarem imagens de ressonância magnética, observaram alterações em ligações funcionais nas redes cerebrais no que se refere à função executiva. 
Tabela 2

Métodos utilizados para avaliação das funções executivas.

\begin{tabular}{lccccccc}
\hline Teste & $\%$ & Teste & $\%$ & Teste & $\%$ & Teste & $\%$ \\
\hline Shifting Atention & 5,56 & GDT & 5,56 & PAOFI & 5,56 & D-KEFS & 11,11 \\
CFQ & 5,56 & COWAT & 5,56 & NAB & 5,56 & WCST & 16,67 \\
GRC & 5,56 & WFT & 5,56 & MMQ & 5,56 & Digit Span & 22,22 \\
VWMT & 5,56 & Category Fluency & 5,56 & Trail making & 11,11 & BRIEF & 22,22 \\
IGT & 5,56 & N-back & 5,56 & VFT & 11,11 & Stroop & 50,00 \\
\hline
\end{tabular}

CFQ: Cognitive Failures Questionnaire; GRC: Global Rating of Cognition; VWMT: Verbal Working Memory Task; IGT: Iowa Grambling Task; GDT: Game of Dice Task; COWAT: Controlled Oral Word Association Test; WFT: Word Fluency Test; N-back test of working memory; PAOFI: Patient's Assessment of Own Functioning Inventory; NAB: Neuropsychological Assessment Battery categories test, MMQ: Multifactorial Memory Questionnaire Ability Scale VFT: Verbal Fluency Test; D-KEFS: Dellis-Kaplan Executive Functions System; WCST: Wisconsin Card Sorting Test; BRIEF: Behavior Rating Inventory of Executive Function.

Tabela 3

Componentes das funções executivas abordadas nos estudos levantados.

\begin{tabular}{lcc}
\hline Componente & Número de estudos & $\%$ \\
\hline Impulsividade/Controle inibitório & 9 & 50,0 \\
Fluência & 8 & 44,4 \\
Componentes gerais & 8 & 44,4 \\
Memória operacional & 6 & 33,3 \\
Flexibilidade cognitiva & 5 & 27,8 \\
Categorização & 4 & 22,2 \\
Tomada de decisão & 1 & 5,6 \\
Planejamento & 0 & 0
\end{tabular}

Também existiram estudos os quais não afirmam ter encontrado diferenças significativas em resultados de testes em grupos de mulheres com câncer de mama com quimioterapia e demais grupos como grupo de mulheres saudáveis ou de mulheres com câncer de mama sem quimioterapia (Ando-Tanabe, 2014, Chen et al., 2013; Kam et al., 2016), apesar de um estudo encontrar reduções de atividades cerebrais por meio de neuroimagens (Kam et al., 2015).

Algumas pesquisas levantadas destacaram determinadas alterações do funcionamento executivo as quais tiveram relação significativa direta entre comorbidades como fadiga (Askren et al., 2014), depressão (Ando-Tanabe, 2014; Ganz et al., 2003) e outras condições como recebimento da terapia hormonal (Ganz et al., 2003). Todavia, a correlação com a depressão somente não foi corroborada por Koppelmans (2012).
Finalmente, ressalta-se que, alguns estudos verificaram que houve melhoras do funcionamento executivo após a conclusão da quimioterapia (Ando-Tanabe, 2014; Chen et al., 2014; Dumas, 2013; Jansen, 2011; Lyon et al., 2016; Park et al., 2015; Zheng et al., 2014), sendo mais lenta em mulheres com idade mais avançada e de escolaridade baixa (Kesler et al., 2011; Zheng et al., 2014).

\section{Discussão}

De antemão, ressalta-se que os componentes relacionados às funções executivas se referem a planejamento, controle inibitório, tomada de decisão, flexibilidade cognitiva, memória operacional, categorização e fluência (Malloy-Diniz et al., 2014). E, embora a avaliação neuropsicológica não tenha o propósito de conhecer qualquer tipo de função pura- 
mente, de acordo com Hamdan e Pereira (2009), as interconexões cerebrais das funções executivas têm sido evidenciadas por estudos de neuroimagem, neurofisiologia e neuropsicologia, demonstrando sua relação com diferentes áreas corticais e subcorticais. Uma avaliação neuropsicológica das funções executivas, por sua vez, pode auxiliar no diagnóstico clínico, na compreensão do perfil cognitivo do indivíduo bem como construir com o prognóstico, reabilitação, acompanhamento farmacológico e psicossocial.

Poucos artigos encontrados nessa revisão dedicaram-se à avaliação neuropsicológica para investigação exclusiva de aspectos das funções executivas (Kam et al., 2016, Miao et al., 2016, McDonald et al., 2013). Como demonstrado, a grande maioria apenas limita-se ao exame de poucos aspectos associados ao funcionamento executivo, o que pode limitar o rastreio das funções executivas, demonstrando uma necessidade por maior aprofundamento do tema (Lyon et al., 2016; Yao et al., 2016; Park et al., 2015; Zheng et al., 2014; Ganz et al., 2003; Chen X et al., 2014; Koppelmans, 2012; Piccirillo, et al., 2015; Askren et al., 2014; Dumas, 2013; Kesler, 2013; Kesler et al., 2011; Ando-Tanabe, 2014, Chen et al., 2013, Jansen, 2011). Esses estudos, apesar de avaliarem uma diversidade de domínios cognitivos, foram selecionados nesse trabalho por incluíremem instrumentos de avaliação de subdomínios das funções executivas podendo fornecer informações - não menos importantes - sobre o impacto da quimioterapia em pacientes com câncer de mama.

Outra nota a ressaltar, ainda quanto às pesquisas selecionadas, trata-se do local de onde estas partem. Todas foram disponibilizadas pelo banco de dados de caráter internacional. E, ainda, nenhuma delas foi realizada no Brasil, o que aponta uma escassez de estudos nacionais nos últimos cinco anos. Questiona-se, diante disso, como o rastreio cognitivo executivo tem sido considerado na ciência e nos serviços assistenciais à população com câncer de mama no país.

Os estudos encontrados possuem um desenho observacional quantitativo sendo longitudinal prospectivo - em sua grande maioria - e transversal. Os estudos longitudinais contabilizaram, pelo menos, dois pontos de tempos de coleta de dados. Além disso, a maioria das pesquisas utilizam correlação e caso-controle. O objetivo dos métodos não experimentais ou correlacionais, de acordo com Cozby (2009), é descrever o comportamento como ocorre naturalmente registrando respostas, atribuindo-lhes valores numéricos e analisando estatisticamente. Nesse tipo de estudo, levantam-se hipóteses de possíveis relações, já que é difícil determinar qual variável causa a outra.

Todos utilizaram uma amostra de pacientes com câncer de mama do sexo feminino a partir do estágio inicial da doença. As idades das participantes incluídas no estudo variaram bastante, sendo o mínimo de 18 anos e, o máximo de 80 anos. Isso pode apontar que o quesito de idade foi pouco elaborado como critério de inclusão e exclusão das participantes das pesquisas, talvez pelo caráter de conveniência e facilidade de acesso ao abranger um maior número de pacientes. Segundo Hamdan e Pereira (2012), o quesito da idade deve ser cuidadosamente considerado numa avaliação neuropsicológica tanto durante a escolha dos procedimentos a serem utilizados quanto durante a integração dos resultados, pois estudos têm mostrado uma relação entre o desenvolvimento e as funções executivas, as quais parecem concluir seu processo de maturação no início da idade adulta e declinar no início da velhice.

Outra característica dos estudos também diversificada foi o número das amostras. As quantidades das participantes variaram entre nove a 189, o que pode apontar pouco consenso nos estudos ou diferentes representatividades. Nenhum deles trabalhou com amostragem probabilística, ou seja, quando a probabilidade da amostra reflete exatamente o valor real da população (Cozby, 2009). Isso pode demonstrar a preferência em estabelecer relações entre o funcionamento cognitivo e a quimioterapia em pacientes com câncer de mama, uma das razões para usar amostra não probabilística por conveniência. Nesse caso, estuda-se mais relações entre variáveis a estimativas acuradas de valores da população (Cozby, 2003).

No que se refere à condição do tratamento das participantes, grande parte dos estudos realizou avaliações antes do início da quimioterapia, utilizando os resultados destas como linha de base para a investigação. No entanto, os intervalos para avaliações posteriores variaram, o que também apresentam pouco consenso metodológico. Por exemplo, enquanto algumas utilizaram intervalos após um mês do início da primeira quimioterapia, outras utilizaram intervalos de alguns anos e até décadas.

Essas pesquisas, em geral, tiveram como objetivo explorar condições cognitivas de mulheres com câncer de mama que vivenciaram a quimioterapia, seja 
especificamente das funções executivas (foco desse trabalho) ou de funções cognitivas gerais (maioria dos estudos). Além disso, algumas também buscaram investigar possibilidades correlacionais entre tais condições a outros sintomas prejudiciais interferentes como depressão, ansiedade, fadiga, preocupação e outros dados clínicos.

Para atingir seus objetivos, vários foram os métodos e instrumentos utilizados. Em geral, foram adotados métodos de entrevista e uso de instrumentos de autorrelato sobre o funcionamento cognitivo. No que se refere ao uso de testes, enquanto alguns adotaram apenas uma bateria de testes neuropsicológicos, outros estudos, além destes instrumentos, acrescentaram o uso de técnica de neuroimagem com intuito de revelar anormalidades neurobiológicas cognitivas potencialmente prejudiciais subjacentes à quimioterapia. Porém, cabe destacar, a maior parte dos estudos que usou imagens de ressonância magnética, limitou-se a uma pouca quantidade de testes (usando um ou dois). Nos demais estudos, também foram utilizadas escalas e testes psicológicos, por meio dos quais averiguava-se estados como ansiedade, depressão e fadiga.

A maioria dos métodos utilizados nas pesquisas, inclusive, não foram semelhantes entre si, demonstrando uma ausência de padronização. Segundo Untura e Rezende (2012), o que pode refletir a grande diversidade de métodos encontrados na literatura decorre de o fato da constatação da saúde cognitiva não ser prioridade nos serviços assistenciais. Para elas, a diversidade metodológica dificulta a comparação e a reprodução dos resultados nos serviços.

A escolha dos instrumentos neuropsicológicos para avaliação das funções executivas também variou na realização das pesquisas levantadas. Malloy-Diniz et al. (2014) listam os instrumentos que são geralmente utilizados para avaliar as funções executivas: baterias fixas como Behavior Assessment of the Dysexcutive Syndrome (Bades) e Dellis-Kaplan Executive Functions System (D-KEFS) - para examinar as funções executivas em geral; baterias flexíveis incluindo instrumentos como Teste da Torre de Londres e Torre de Hanói, Teste dos Labirintos na Escala de Wechesler de Inteligência e de Porteous - para examinar planejamento; Stroop, Go-No-Go, Tarefa de Performance Contínua (CPT) e Teste dos Cinco Dígitos - para examinar o controle inibitório; Iowa Grambling Task (IGT) e Tarefa dos dados - para examinar tomada de decisão; Teste das Trilhas, Teste da Seleção de Cartas de Winsconsin - para examinar flexibilidade cognitiva; Provas de repetição de dígitos, Paced Auditory Serial Adidition Test (Pasat), Teste dos Trigramas, Span de Dígitos e Teste dos Cubos de Consi - para examinar memória operacional; Teste de Wechsler de Inteligência e Teste das 20 perguntas - para examinar categorização; e Teste dos Cinco Pontos - para examinar fluência (Malloy-Diniz et al., 2014).

Dos estudos selecionados nessa revisão, somente Kesler et al. (2013) utilizou baterias fixas (como as supracitadas). $\mathrm{O}$ restante dos estudos, personalizaram a bateria de testes que utilizam para explorar as funções executivas escolhendo testes diversos conforme próprios critérios de pesquisa. Ressalta-se que o teste de maior utilização foi Stroop Test. Este propõe-se a observar a dificuldade de processar informações com significados conflitantes simultaneamente e pode oferecer medidas importantes sobre o controle inibitório. Sendo assim, as diferenças metodológicas existentes em grande parte dos estudos levantados acerca do funcionamento executivo, conforme Jurado e Rosselli (2007), dificultam e limitam a generalização dos resultados.

As pesquisas levantadas não assumiram com clareza a perspectiva teórica utilizada, gerando, assim, uma incompreensão acerca de seu referencial, além de impossibilitar comparações entre as mesmas. São muitos os modelos teóricos que definem as funções executivas, o que pode refletir os múltiplos modos de classificá-las e examiná-las, como visto nos estudos levantados. Malloy-Diniz et al. (2014) descrevem que vários modelos explicam o funcionamento executivo. $\mathrm{O}$ conceito de unidade 3 ou unidade executiva de Luria e o modelo de memória operacional de Baddeley e Hich consideram-nas enquanto um único construto. Já outros modelos consideram-nas enquanto processos relativamente independentes os quais interagem entre si, como se fosse um termo guarda-chuva que articula processos cognitivos como memória operacional (ou memória de trabalho), planejamento, solução de problemas, tomada de decisão, controle inibitório, fluência, flexibilidade cognitiva e categorização (Hamdan, \& Pereira, 2009).

Conforme Hamdan e Pereira (2009), um dos teóricos que defendem esse modelo é Barkley que dá ênfase ao controle inibitório e sua integração com a memória operacional, a fala internalizada (autoinstrução, definição e orientações de regras, raciocínio moral e reflexivo sobre o próprio comportamento em curso), a autorre- 
gulação (motivacional e afetiva) e a reconstituição (fluência, criatividade, ensaios mentais, análise e síntese comportamental). Lezak, Howieson, Bigler e Tranel (apud Hamdan, \& Pereira, 2009) indicam que as funções executivas decorrem de processos de etapas sucessivas e interdependentes, a saber: volição (relativo ao comportamento intencional, envolve o estabelecimento de objetivos e a motivação de iniciativa resolutiva); planejamento (identificação de etapas a alcançar e de elementos necessários para tanto); ação propositada (transição para o comportamento em si); e autorregulação comportamental e monitoração do desempenho efetivo (avaliação e flexibilidade de mudança de estratégia).

Demais teóricos, como Bechara e Van Der Linder (2005 apud Malloy-Diniz et al., 2014), Stuss (2011 apud Malloy-Diniz et al., 2014), Fuster (2008 apud Malloy-Diniz et al., 2014) e Happaney e Zelazo (2004 apud Malloy-Diniz et al., 2014), enfatizam dados advindos de correlações anatomoclínicas e respectivos circuitos cerebrais. Nesse sentido, Zelazo e Muller (2002 apud Malloy-Diniz et al., 2014), fracionaram as funções executivas em dois tipos: "frias" - referentes a processos cognitivos como categorização, flexibilidade, fluência verbal com maior atividade no córtex pré-frontal dorsolateral - e "quentes" - aspectos emocionais e motivacionais com maior atividade cortical pré-frontal orbitofrontal. Por fim, outro modelo, de Miyake e colaboradores (2000 apud (Malloy-Diniz et al., 2014), propõem que as funções executivas são compostas por três núcleos - memória operacional, inibição e flexibilidade cognitiva - sendo base para o desempenho de outros componentes mais complexos - solução de problemas, planejamento e raciocínio abstrato.

Apesar do uso de diversos meios para avaliar componentes das funções executivas, como visto, a maioria dos estudos compartilharam conclusões acerca do baixo desempenho do grupo de mulheres com câncer de mama que se receberam a quimioterapia. Todavia, questiona-se quanto à falta de replicação desses estudos, diante da tamanha variabilidade nos métodos de avaliação. Portanto, parece sugestiva a realização de novas pesquisas que contemplem demais componentes e compartilhem métodos de modo a possibilitar comparação dos resultados.

\section{Considerações finais}

As publicações levantadas nesse trabalho apresentaram variações desde a caracterização da amostra até metodologia. Por outro lado, os estudos convergem em grande parte de seus resultados, compartilhando dados relevantes e próximos sobre o funcionamento executivo durante a quimioterapia.

Entretanto, essas informações ainda são insuficientes e exigem um maior aprofundamento na investigação sobre o tema no que se refere às funções executivas. Subdomínios das funções executivas precisam ser investigados com maior integralidade, incluindo a todos, a fim de obter um maior panorama funcional. Sugere-se novos estudos acerca do rastreio das funções executivas, tão importantes para o desempenho emocional e social durante o processo do tratamento e manutenção da saúde na vida dessas mulheres.

\section{Referências}

Ahles, T. A., \& Saykin, A. J. (2007). Candidate mechanisms for chemotherapy-induced cognitive changes. Nature Reviews Cancer, 7(3), 192-201. https://doi.org/10.1038/nrc2073

Ando-Tanabe, N., Iwamitsu, Y., Kuranami, M., Okazaki, S., Yasuda, H., Nakatani, Y. et al. (2014). Cognitive function in women with breast cancer receiving adjuvant chemotherapy and healthy controls. Breast Cancer (Tokyo, Japan), 21(4), 453-462. https://doi.org/10.1007/s12282-012-0405-7

Askren, M. K., Jung, M., Berman, M. G., Zhang, M., Therrien, B., Peltier, S. et al. (2014). Neuromarkers of fatigue and cognitive complaints following chemotherapy for breast cancer: a prospective fMRI investigation. Breast Cancer Research and Treatment, 147(2), 445-455. https://doi.org/10.1007/s10549-014-3092-6

Caponeto, R., Lange, L. M. (2008). Quimioterapia. In: V. A. Carvalho, M. H. P. Franco, M. J. Kovács, R. P. Liberato, R. C. Macieira, M. T. Veit et.al. Temas em Psico-Oncologia (pp. 155-67). São Paulo: Summus.

Chen, X., Li, J., Zhu, C., Li, D., Zhang, J., \& Wang, K. (2014). [Cognitive function in breast cancer patients on chemotherapy: a longitudinal study]. Zhonghua Yi Xue Za Zhi, 94(1), 27-30.

Chen, X., Zhu, X., Li, J., Qiu, L., Zheng, L., Yu, F. et al. (2013). Dissociation of decision making under ambiguity and decision making under risk in breast cancer patients receiving adjuvant chemotherapy: a neuropsychological study. Brain Research, 1533, 63-72. https://doi.org/10.1016/j.brainres.2013.08.015 
Cozby, P. C. (2003). Métodos de pesquisa em ciências do comportamento. São Paulo: Atlas.

Dumas, J. A., Makarewicz, J., Schaubhut, G. J., Devins, R., Albert, K., Dittus, K., \& Newhouse, P. A. (2013). Chemotherapy altered brain functional connectivity in women with breast cancer: a pilot study. Brain Imaging and Behavior, 7(4), 524-532. https://doi.org/10.1007/s11682-013-9244-1

Ganz, P. A., Kwan, L., Castellon, S. A., Oppenheim, A., Bower, J. E., Silverman, D. H. S. et al. (2013). Cognitive complaints after breast cancer treatments: Examining the relationship with neuropsychological test performance. Journal of the National Cancer Institute, 105(11), 791-801. https:// doi.org/10.1093/jnci/djt073

Hamdan, A. C., \& Pereira, A. P. A. (2009). Avaliação neuropsicológica das funções executivas: considerações metodológicas. Psicologia: Reflexão e Crítica, 22(3): 386-393. https://doi.org/10.1590/S0102-79722009000300009

Howieson, D. B., Lezak, M. D. (2014). A avaliação neuropsicológica. In: S. C. Yudofsky, \& E. Robert. Fundamentos de neuropsiquiatria e ciência do comportamento (2a ed., pp. 41-66). Porto Alegre, RS: Artmed.

Instituto Nacional de Câncer. (2012). Ensino em atenção oncológica no Brasil:carênciase oportunidades. Rio de Janeiro, RJ: Inca. Recuperado de http://wwwl.inca.gov.br/inca/Arquivos/ensino_em_atencao_oncologica_no_brasill.pdf

Instituto Nacional de Câncer. (2016). Estimativas 2014: incidência de câncer no Brasil. Rio de Janeiro, RJ: Inca. Recuperado de http://www.inca.gov.br/bvscontrolecancer/publicacoes/Estimativa_2014.pdf

Jansen, C. E., Cooper, B. A., Dodd, M. J., \& Miaskowski, C. A. (2011). A prospective longitudinal study of chemotherapy-induced cognitive changes in breast cancer patients. Supportive Care in Cancer, 19(10), 1647-1656. https://doi.org/10.1007/s00520-010-0997-4

Jurado, M. B., \& Rosselli, M. (2007). The elusive nature of executive functions: a review of our current understanding. Neuropsychology Review, 17(3): 213-233. https://doi.org/10.1007/s11065-007-9040-z

Kam, J. W. Y., Boyd, L. A., Hsu, C. L., Liu-Ambrose, T., Handy, T. C., Lim, H. J. et al. (2016). Altered neural activation during prepotent response inhibition in breast cancer survivors treated with chemotherapy: an fMRI Study. Brain Imaging and Behavior, 10(3), 840-848. https://doi.org/10.1007/s11682-015-9464-7

Kesler, S. R., Kent, J. S., \& O'Hara, R. (2011). Prefrontal cortex and executive function impairments in primary breast cancer. Archives of Neurology, 68(11), 1447-1453. https://doi.org/10.1001/archneurol.2011.245

Kesler, S. R., Watson, C., Koovakkattu, D., Lee, C., O’Hara, R., Mahaffey, M. L., \&Wefel, J. S. (2013). Elevated prefrontal myo-inositol and choline following breast cancer chemotherapy. Brain Imaging and Behavior, 7(4), 501-510. https://doi.org/10.1007/s11682-013-9228-1

Koppelmans, V., Breteler, M. M. B., Boogerd, W., Seynaeve, C., Gundy, C., \& Schagen, S. B. (2012). Neuropsychological performance in survivors of breast cancer more than 20 years after adjuvant chemotherapy. Journal of Clinical Oncology, 30(10), 1080-1086. https:// doi.org/10.1200/JCO.2011.37.0189

Lyon, D. E., Cohen, R., Chen, H., Kelly, D. L., Starkweather, A., Ahn, H. C. et al. (2016). The relationship of cognitive performance to concurrent symptoms, cancer- and cancer-treatment-related variables in women with early-stage breast cancer: a 2-year longitudinal study. Journal of Cancer Research and Clinical Oncology, 142(7), 1461-1474. https:// doi.org/10.1007/s00432-016-2163-y

McDonald, B. C., Conroy, S. K., Smith, D. J., West, J. D., \& Saykin, A. J. (2013). Frontal gray matter reduction after breast cancer chemotherapy and association with executive symptoms: a replication and extension study. Brain, Behavior, and Immunity, 30(Suppl), S117-S125. https://doi.org/10.1016/j.bbi.2012.05.007

Malloy-Diniz, L. F., Paula, J. J., Sedó, M., Fuentes, D., Leite, W. B. (2014). Neuropsicologia das funções executivas e da atenção. In D. Fuentes, L. F. Malloy-Diniz, C. H. P. Camargo, \& R. M. Consenza. Neuropsicologia:teoria e prática (2a ed., pp.115-138). Porto Alegre, RS: Artmed.

Miao, H., Li, J., Hu, S., He, X., Partridge, S. C., Ren, J. et al. (2016). Long-term cognitive impairment of breast cancer patients after chemotherapy: a functional MRI study. European Journal of Radiology, 85(6), 1053-1057. https://doi.org/10.1016/j.ejrad.2016.03.011

Park,J.H., Bae, S.H., Jung,Y.S., \& Jung,Y.M. (2015). [Prevalence and characteristics of chemotherapy-related cognitiveimpairmentinpatients with breast cancer]. KoreanAcadNurs, 45(1),118-128. Korean. https://doi.org/10.4040/jkan.2015.45.1.118

Piccirillo, J. F., Hardin, F. M., Nicklaus, J., Kallogjeri, D., Wilson, M., Ma, C. X. et al. (2015). Cognitive impairment after chemotherapy related to atypical network architecture for executive control. Oncology, 88(6), 360-368. https:// doi.org/10.1159/000370117 
Reiriz, A. B. Estudo da função cognitiva em camundongos submetidos ao agente quimioterápico ciclofosfamida (Tese de doutorado). Programa de Pós-Graduação em Medicina, da Universidade Federal do Rio Grande do Sul, Porto Alegre, RS.

Robbins, T. W., Weinberger, D., Taylor, J. G., \& Morris, R. G. (1996). Dissociating executive functions of the prefrontal cortex. Philosophical Transactions of the Royal Society ofLondon. Series B, Biological Sciences, 351(1346), 1463-1470. https://doi.org/10.1098/rstb.1996.0131

Untura, L. P., \& Rezende, L. F. (2012). A função cognitiva em pacientes submetidos à quimioterapia: uma revisão integrativa. Revista Brasileira de Cancerologia, 58(2): 257-265.

Yao, C., Rich, J. B., Tannock, I. F., Seruga, B., Tirona, K., \& Bernstein, L. J. (2016). Pretreatment differences in intraindividual variability in reaction time between women diagnosed with breast cancer and healthy controls. Journal of the International Neuropsychological Society, 22(5), 530-539. https:// doi.org/10.1017/S1355617716000126

Zheng,Y.,Luo, J., Bao,P., Cai, H., Hong,Z., Ding, D. etal. (2014). Long-term cognitivefunction changeamongbreastcancer survivors. Breast Cancer Research and Treatment, 146(3), 599-609. https://doi.org/10.1007/s10549-014-3044-1

\section{Camila Vasconcelos Carnaúba Lima}

Mestranda em Psicologia pela Universidade Federal de Alagoas, Maceió, AL, Brasil.

E-mail: camila_carnauba@hotmail.com

\section{Raner Miguel Ferreira Póvoa}

Docente do Programa de Pós-graduação em Psicologia da Universidade Federal de Alagoas, Maceió, AL, Brasil.

E-mail: rpovoaraner05@gmail.com

Endereço para envio de correspondência:

Universidade Federal de Alagoas, ICHCA.

Campus A.C. Simões v. Lourival Melo Mota, s/n, , CEP: 57072970. Tabuleiro do Martins- Maceio, AL - Brasil. Telefone: (82) 32141336

Recebido 29/12/2016

Revisado 16/07/2017

Aprovado 30/08/2017

Received $12 / 29 / 2016$

Reformulated $07 / 16 / 2017$

Approved 08//302017

Recibido 29/12/2016

Reformulado 16/07/2017

Aceptado 30/08/2017

Como citar: Lima, C.V.C., Póvoa, R.M.F (2017). Mulheres Submetidas à Quimioterapia e suas Funções Cognitivas. Psicologia: Ciência e Profissão, 37(4), 970-980. https://doi.org/10.1590/1982-3703004772016

How to cite: Lima, C.V.C., Póvoa, R.M.F (2017). Women Undergoing Chemotherapy and Their Cognitive Functions. Psicologia: Ciência e Profissão, 37(4), 970-980. https://doi.org/10.1590/1982-3703004772016

Cómo citar: Lima, C.V.C., Póvoa, R.M.F (2017). Mujeres Sometidas a Quimioterapia y sus Funciones Cognitivas. Psicologia: Ciência e Profissão, 37(4), 970-980. https://doi.org/10.1590/1982-3703004772016 